\title{
A Further Look at Therapeutic Interventions for Suicide Attempts and Self-Harm in Adolescents: An Updated Systematic Review of Randomized Controlled Trials
}

\author{
Udita lyengar ${ }^{1 *+}$, Natasha Snowden ${ }^{1 \dagger}$, Joan R. Asarnow ${ }^{2}$, Paul Moran ${ }^{3,4}$, Troy Tranah ${ }^{5}$ and \\ Dennis Ougrin ${ }^{1,6}$ \\ ${ }^{1}$ Department of Child and Adolescent Psychiatry, Institute of Psychiatry, Psychology, and Neuroscience (IoPPN), King's \\ College London, London, United Kingdom, ${ }^{2}$ Semel Institute of Neuroscience and Behavior, David Geffen School of Medicine, \\ University of California, Los Angeles, Los Angeles, CA, United States, ${ }^{3}$ National Institute for Health Research Bristol \\ Biomedical Research Centre, University Hospitals Bristol NHS Foundation Trust and University of Bristol, Bristol, United \\ Kingdom, ${ }^{4}$ Department of Population Health Sciences, Centre for Academic Mental Health, Bristol Medical School, \\ University of Bristol, Bristol, United Kingdom, ${ }^{5}$ Institute of Psychiatry, Psychology, and Neuroscience (IoPPN), London, \\ United Kingdom, ${ }^{6}$ South London and Maudsley National Health Service (NHS) Foundation Trust, London, United Kingdom
}

\section{OPEN ACCESS}

Edited by:

Yossi Levi-Belz,

Ruppin Academic Center, Israel

Reviewed by:

Paul William George Surgenor

Pieta House, Ireland

Sami Hamdan,

Hungarian Academy of Sciences

(MTA), Hungary

*Correspondence:

Udita lyengar

udita.iyengar@kcl.ac.uk

†These authors share first authorship

Specialty section:

This article was submitted to

Psychopathology,

a section of the journal

Frontiers in Psychiatry

Received: 19 December 2017 Accepted: 24 October 2018 Published: 23 November 2018

Citation:

lyengar U, Snowden N, Asarnow JR,

Moran $P$, Tranah $T$ and Ougrin $D$ (2018) A Further Look at Therapeutic Interventions for Suicide Attempts and Self-Harm in Adolescents: An Updated Systematic Review of Randomized Controlled Trials.

Front. Psychiatry 9:583. doi: 10.3389/fpsyt.2018.00583
Background: Suicide attempts (SA) and other types of self-harm (SH) are strong predictors of death by suicide in adolescents, emphasizing the need to investigate therapeutic interventions in reduction of these and other symptoms. We conducted an updated systematic review of randomized controlled trials (RCTs) from our previous study reporting therapeutic interventions that were effective in reducing $\mathrm{SH}$ including SA, while additionally exploring reduction of suicidal ideation (SI) and depressive symptoms (DS).

Method: A systematic literature search was conducted across OVID Medline, psycINFO, PubMed, EMBASE, and Cochrane Library from the first available article to October 22nd, 2017, with a primary focus on RCTs evaluating therapeutic interventions in the reduction of self-harm. Search terms included self-injurious behavior; self-mutilation; suicide, attempted; suicide; drug overdose.

Results: Our search identified 1,348 articles, of which 743 eligible for review, yielding a total of 21 studies which met predetermined inclusion criteria. Eighteen unique therapeutic interventions were identified among all studies, stratified by individual-driven, socially driven, and mixed interventions, of which 5 studies found a significant effect for primary outcomes of self-harm and suicide attempts (31.3\%), and 5 studies found a significant effect for secondary outcomes of suicidal ideation and depressive symptoms (29.4\%) for therapeutic intervention vs. treatment as usual. Collapsing across different variations of Cognitive Behavior Therapy (CBT), and classifying Dialectical Behavior Therapy for Adolescents (DBT-A) as a type of CBT, CBT is the only intervention with replicated positive impact on reducing self-harm in adolescents.

Conclusion: While the majority of studies were not able to determine efficacy of therapeutic interventions for both primary and secondary outcomes, our systematic review suggests that individual self-driven and socially-driven processes appeared to show the greatest promise for reducing suicide attempts, with benefits of combined 
self-driven and systems-driven approaches for reducing overall self-harm. Further RCTs of all intervention categories are needed to address the clinical and etiological heterogeneity of suicidal behavior in adolescents, specifically suicidal ideation and depressive symptoms.

Keywords: suicide, self-harm, NSSI, depression, suicidal ideation, adolescent, RCT

\section{INTRODUCTION}

Suicide is a major global and public health concern (1). It is the second leading cause of death in people age 15-24 years (2) and there is a pressing need to identify effective interventions to reduce the risk of suicide. Non-fatal suicide attempts (SA) can be defined as self-directed injuries with implicit or explicit intent to kill oneself, while non-suicidal self-injury (NSSI) is direct destruction of one's body without intention to die (3). For the latter, it is useful to consider the definition of nonsuicidal self-injury found in the DMS-5, which states that the preoccupied individual partakes in premeditated, self-directed damage to themselves in order to relieve negative experiences and does not exhibit suicidal intent through this behavior (4). Both suicide attempts (SA) and the broader category of "self-harm" (SH, which includes non-suicidal self-injury) are among the strongest predictors of death by suicide (5-7), and have therefore appropriately been the focus of therapeutic interventions for adolescents to decrease risk of suicide.

There has been significant progress in detection (8), identifying subtypes, understanding the long-term outcomes (9), and understanding help-seeking in adolescents with SH (10). There has also been recent progress regarding the treatment of self-harm in adolescents. We conducted the first metaanalysis of randomized controlled trials (RCTs) to specifically evaluate therapeutic interventions (TIs) in reducing $\mathrm{SH}$ in adolescents (11). A significant effect was found for tested interventions reducing $\mathrm{SH}$ compared to treatment as usual (TAU). While results evaluating the effects of therapeutic interventions on NSSI were generally consistent with those for overall self-harm, the effect size was weaker and escaped statistical significance. In contrast, there was little to no evidence of benefits of tested interventions in reducing suicide attempts. Our findings highlighted both the beneficial effects of therapeutic interventions for self-harm as a global category, the challenges of reducing the risk of future suicide attempts and the need for rigorous and replicable studies.

In addition to self-harm and suicide attempts, however, depression in adolescence is another key contributor to suicidal behavior $(12,13)$. A recent National Confidential Inquiry into Suicide and Homicide by People with Mental Illness (NCISH) report of suicide deaths in England and Wales between 2014 and 2015 indicated that out of 285 suicide deaths that occurred in youths aged $10-20,52 \%$ had a history of $\mathrm{SH}$, while $58 \%$ expressed thoughts of suicide or hopelessness (14). Depressive symptoms themselves have been found to be significant and independent contributors to elevated levels of deliberate self-harm in young people (15-18). Therefore, it would appear that the reduction of depressive symptoms and suicidal ideation (19) may be an important mechanism underpinning the effectiveness of certain treatments for suicide prevention.

We therefore sought to extend and update our initial metaanalysis focused on the reduction of self-harm and suicide attempts, while also examining the effect of a variety of unique therapeutic interventions on levels of depressive symptoms and suicidal thoughts. In this way, we acknowledge the relevance of these mechanisms to the field and aim to advance our previous study findings with a wider criterion and selection of interventions. Our primary outcomes were the reduction in self-harm including NSSI or suicide attempts (SAs), and our secondary outcomes were the reduction in suicidal ideation (SI), meaning thoughts and feelings related to suicide, as well as depressive symptoms (DS).

\section{METHODS}

\section{Eligibility and Selection}

We followed the same methodology as our previous systematic review [see (11)], using "self-harm" as an encompassing term, including previous suicide attempts, non-suicidal self-injury, and deliberate self-harm with undetermined intent. However, in addition to the original systematic review's reduction of self-harm and suicide attempts (primary outcomes) we also examined decrease of suicidal ideation and depressive symptoms (secondary outcomes) as markers of the efficacy of the therapeutic interventions.

\section{Inclusion Criteria}

Inclusion to the update depended on: study type, sample age, and frequency of self-harm occurring within the sample. We included only studies which were clinical, randomized trials of therapeutic interventions, defined as any theoretically coherent, manualized, psychological, psychosocial, or pharmacological intervention, compared to a placebo or control treatment (11). Further, we included only studies with a majority (>50\%) child and adolescent population ( $<18$ years old), engaged in either selfharm or had attempted suicide. Studies from all countries and languages were considered eligible, if they were accompanied by an English abstract.

\section{Exclusion Criteria}

Potential studies were excluded from the update if self-harm was a symptom of an overarching developmental condition (i.e., autism or intellectual disability). Finally, studies that did not meet the threshold score of $>2$ on the Jadad quality assessment tool (20), specifically used to ascertain methodological quality, coherence to blinding and allocation procedures, and amenability to participant attrition, were excluded from consideration. 


\section{Literature Search Strategy}

A literature search was conducted through standard online databases (OVID Medline, psycINFO, PubMed, EMBASE, and Cochrane Library) in order to identify RCTs evaluating the efficacy of therapeutic interventions for adolescents with selfharm against control conditions. To maintain consistency with our first study, the same inclusion and exclusion criteria were utilized in this update. We excluded studies in which self-harm occurred as a result of stereotypic self-injurious behaviors such as those seen in moderate to severe forms of neurological disorders (e.g., Autism, Intellectual Disability), due to the complex neurological mechanisms which underlie the self-harm not otherwise seen outside of these conditions. All the aforementioned databases were searched from the first available article until October $22^{\text {nd }}, 2017$. Consistent with our original methodology, the following subject headings or $\mathrm{MeSH}$ keywords were used: self-injurious behavior; self-mutilation; suicide, attempted; suicide; drug overdose. When available, filters for study type and participant age were applied, with an additional manualized filter used for psycINFO to specifically identify clinical trials.

\section{RESULTS}

\section{Study Selection}

A total of 1,348 articles were found, with 743 of those studies eligible for review following duplicate removal (Figure 1). The screening procedure consisted of three phases: title, abstract and full text screening, with the latter two conducted independently by the two authors (UI and NS). Title screening was conducted by NS as a preliminary measure to ensure the exclusion of any notpertinent studies and duplicates, reducing the number of eligible studies from 743 to 102 . Within the abstract screening phase, 31 articles were eliminated as they failed to meet any one aspect of the inclusion criteria, producing a yield of 71 articles for full text screening. In the third and final phase, we excluded 50 studies, 16 of which had a sample with a mean age above 18 years, 23 which did not have a majority of adolescents who engaged in self-harming behaviors, 9 which were not RCTs, 1 which has been replaced by a paper with more recent follow-up data, and 1 which did not meet quality standards. We chose to remove one study included in the original systematic review (21), as its investigation of a treatment designed to increase linkage to outpatient services did not assess the same outcomes which we are addressing in this update. Disagreements in any phase of the screening process were resolved by consensus discussion between the authors (UI and NS).

\section{Therapeutic Interventions}

The final selection of 21 studies contained 18 unique therapeutic interventions. Two of the final 21 studies $(22,23)$ were replication trials assessing the efficacy of Developmental Group Psychotherapy (24), while another paper (25) was a follow-up to a previous pilot trial of Emotion Training Regulation (ERT) (26). As such, 18 unique interventions were identified among all studies. To facilitate analysis, the interventions can be stratified and evaluated by the underlying theoretical principles, including self-driven cognitive, behavioral and regulatory interventions (referred to as self-driven) and interventions which require engagement with family and social support (referred to as socially-driven). A brief description of the interventions and the study origins are listed in Table 1, with self-driven and socially-driven components identified for each respective study in Table 2. Results below are reported first by interventions which we believed had a primarily self-driven focus, followed by those with a primarily socially-driven focus, and finally, interventions which demonstrated aspects of both self-driven and sociallydriven principles.

\section{Primary Outcome Assessment}

Table 3 provides a brief summary of primary outcomes of the studies for comparison of therapeutic intervention examined, and those that had significant group differences, significant overall differences, or null or negative findings. Of the 21 studies examined, 16 studies explored primary outcomes, out which $5(31.3 \%)$ found significant group differences for intervention vs. treatment as usual $(24,28,31,32,42)$, across all types of treatments.

\section{Significant Differences in Therapeutic Interventions vs. Treatment as Usual}

We first examined interventions that addressed individual problem solving, mentalization, cognitive behavior or skills deficits [these included treatments such as Cognitive Behavioral Therapy [CBT], Mentalization Based Therapy [MBT], Dialectical Behavioral Therapy [DBT]]. The only identified studies with a purely self-driven intervention model were those that evaluated Developmental Group Psychotherapy, an intervention which used cognitive-behavioral, problem-solving, dialectical, and psychodynamic group psychotherapy strategies. In a small initial trial among adolescents with repeated SH referred to child and adolescent mental health services in the UK, Developmental Group Psychotherapy compared to treatment as usual was associated with a significantly lower risk of repeating self-harm, with a lower latency period for repeated attempts, indicating an absolute risk reduction of $26 \%$ (24). The authors cautioned that this strong effect was likely due to a smaller sample, and urged replication studies with a larger sample. Indeed, two efforts at replication failed to find a significant advantage for the Developmental Group Psychotherapy intervention. These trials included one conducted in Australia with supervision from the original UK development team and somewhat different sampling criteria: youths referred for general child and adolescent mental health services identified with repeat self-harm (22). The other trial was a large trial $(N=366)$ conducted in the UK with members of the original development team and also failed to find an advantage for the Developmental Group Psychotherapy (23) over treatment as usual.

One study conducted in Australian outpatient mental health clinics used a socially driven intervention to evaluate a strengthbased family education program, called Resourceful Adolescent Parent Program (RAP-P). Among patients recruited suicidal adolescents from emergency departments or public mental health services, the RAP-P program resulted in a significant improvement on a 9-item suicide index assessing suicide 


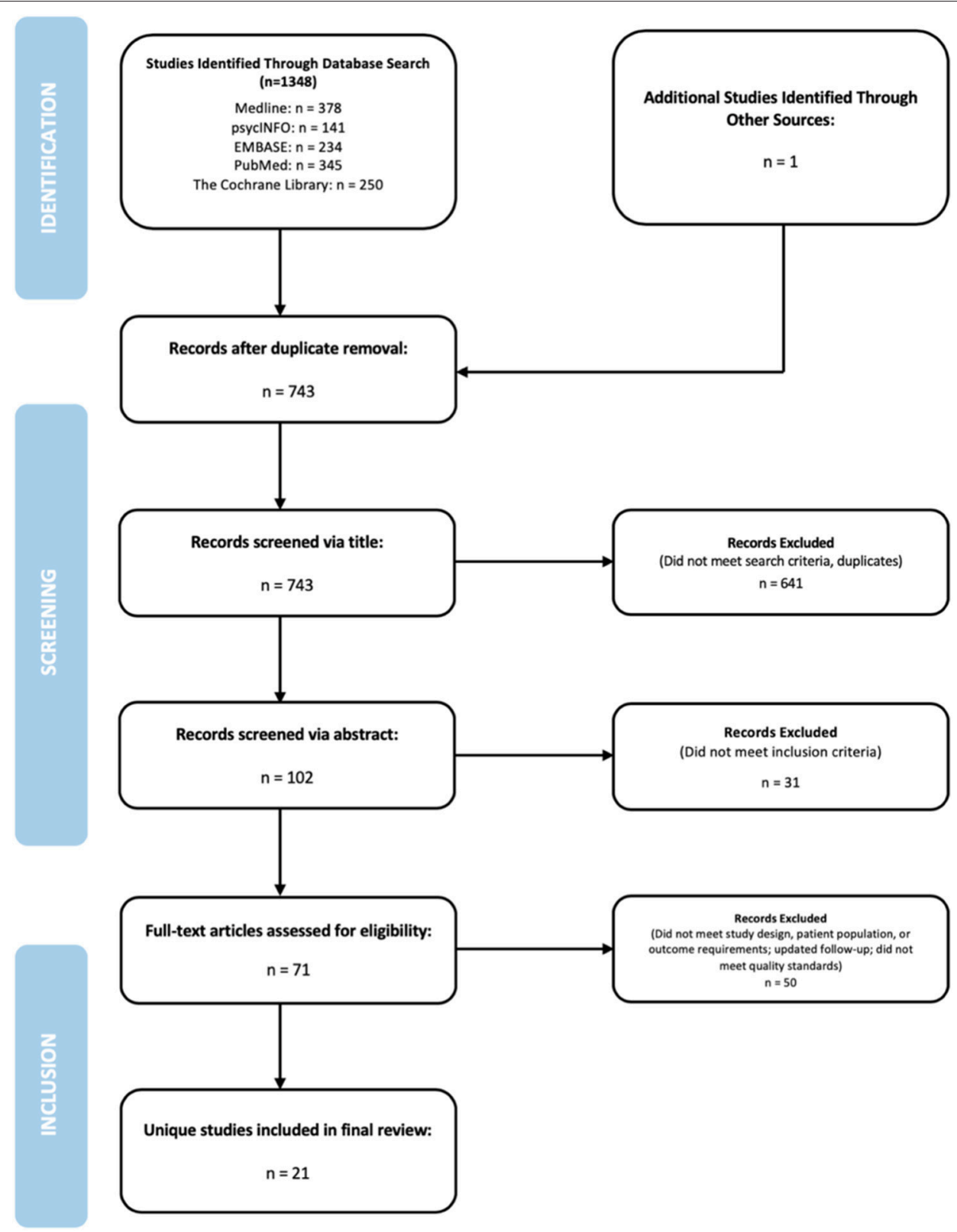

FIGURE 1 | Study flow diagram.

ideations, plans, threats, self-harm, and suicide attempts both at the 3-month post-treatment point, and at a 6-month followup compared to treatment as usual (41). This study was the only exclusively socially-driven intervention model to find a significant group difference on primary outcomes and it should be noted that the outcome variable was a broad measure of suicidality rather than a measure specifically of self-harm or suicide attempts.

Four trials examined more combined self-driven and system driven approaches. We begin with Mentalization-Based Treatment for adolescents (MBT-A), a manualized one-year psychodynamic psychotherapy rooted in attachment theory. MBT-A has a strong self-driven component consisting of weekly individual MBT-A sessions and compared to the other approaches in the combined approach group, the weakest of the socially-driven components; specifically, monthly family Mentalization-based family therapy. In an initial trial, Rossouw and Fonagy (32) found that adolescents selected for the presence of both self-harming behaviors and depression in the MBT- A condition had fewer self-harm episodes over the course of the treatment compared to treatment as usual youths and that the MBT-A group had a higher recovery rate and a reduction of selfharm at the end of the 12-month treatment. Thus, results from this initial trial support the efficacy of MBT-A for reducing overall self-harm. Results specifically for suicidal behavior were not reported. 
TABLE 1 | Descriptions and Study Origins of Therapeutic Interventions of the Selected Randomized Controlled Trials.

\begin{tabular}{lll}
\hline TI name & Study origin & Description \\
\hline CBT-SP & Alavi et al. (27) & $\begin{array}{l}\text { A 3-phase Cognitive-Behavioral Therapy protocol adapted specifically for suicide prevention. Utilizes cognitive } \\
\text { behavioral principles according to the Stanley et al. model, and is comprised of } 12 \text { weekly sessions, the first of } \\
\text { which includes parents. }\end{array}$
\end{tabular}

Integrated Cognitive Behavioral Therapy (I-CBT)

DBT-A

Esposito-Smythers et al. (28)

Mehlum et al. (29)

Developmental Group Wood et al. (24) Psychotherapy

Skills-Based Treatment (SBT)

Emotion Regulation Training (ERT)

Safe Alternative for Teens and Youth (SAFETY)

Mentalization Based

Therapy for

Adolescents (MBT-A)

Cognitive Analytic

Therapy (CAT)

Therapeutic

Assessment

Emergency Tokens

Home-based Family Intervention

Family Intervention for Suicide Prevention

(FISP)

Family-Based Crisis

Intervention

Attachment-Based

Family Therapy (ABFT)

Youth Nominated

Support Team-I

Youth Nominated Support Team-II

Resourceful Adolescent Parent Program (RAP-P)

Asarnow et al. (31) (32)

Chanen et al. (33)

Ougrin et al. (34)

Asarnow et al. (6)

Wharff et al. (37)

King et al. (39)

King et al. (40)
Donaldson et al. (30)

Donaldson et al. (26)

Utilizes cognitive behavioral techniques such as restructuring, problem-solving, affect regulation and communication skills to remediate maladaptive cognitions and provide skills training for the attending adolescent and parents. One-year long intervention consisting of three treatment phases, involving with weekly, bi-weekly, and monthly individual adolescent, family, and parent training sessions.

Streamlined Dialectical Behavioral Therapy protocol adapted for adolescents, incorporating a new skills module to address emotion dysregulation amongst adolescents and their families. Nineteen-week long intervention involving weekly individual therapy, multi-family skills training, and family therapy sessions.

Integrates techniques from problem solving and cognitive and dialectical behavioral interventions to resolve issues around relationships, school problems, peer relationships, depression and self-harm, hopelessness, and feelings about the future. Delivered through a maximum of 19 acute and long-term group sessions run in tandem, administered by a variety of trained personnel.

Intervention designed to target problem solving and affect management skills in self-harming adolescents through cognitive behavioral strategies such as restructuring and relaxation. Delivered by therapists trained in SBT for an undefined number of sessions (mean number of sessions: 9.25).

Designed to teach participants ways of coping with affective instability, daily stressors, and psychological vulnerability through psychoeducation and behavior modification. Treatment is conducted through 17 weekly multi-phase group sessions delivered by therapists trained in ERT.

Emergency Department (ED) family-centered intervention informed by CBT and DBT aimed to reduce future suicide attempts by strengthening protective supports, teaching skills for managing stress reactions and formulating strategies for creating a safe environment for the adolescent. Treatment is conducted through 12 weekly individual and joint sessions for adolescents and their parents, delivered by two therapists, each working with the family.

Rossouw and Fonagy An adapted form of Mentalization-based Treatment, a manualised intervention focusing on impulsivity and affect regulation, helping to enhance the patient's understanding of how to represent feelings in emotionally challenging situations. Year-long intervention with weekly individual and family based therapy (MBT-F) delivered by trained therapists.

Time limited, integrated model of development and psychopathology, equipping the patient with tools more effectively manage stressful situations which could lead to a repetition of pathological behavior. Conducted through 24 weekly sessions delivered by therapists trained in CAT.

Cotgrove et al. (35)

Harrington et al. (36)

Diamond et al. (38)

Manualised assessment protocol for self-harming adolescents facilitating the identification of the target problem, enhancing motivation for change, and exploring ways of relieving vicious cycles. Assessment takes place in one session and is delivered by a trained clinician.

Self-harming adolescents were allotted a token allowing hospital re-entry without question, to be used when adolescent was in need of escaping an intolerable (family) environment.

Short-term, intensive, focused, action orientated intervention used to address family dysfunction without lengthy treatment commitments or the need to present to a hospital setting. Conducted by psychiatric social workers during 5 home-based therapy sessions.

Brief ED intervention which focuses on building a collaboration between adolescents and their parents by identifying and addressing the causes, reaction, and future actions related to the committed suicide attempt. Administered by a trained clinician.

Brief intervention which provides the family with tools to manage current and future crises through psycho-education, cognitive behavioral skill building, therapeutic readiness and safety planning. Delivered during the adolescent's visit to the ED by a research clinician.

Designed to improve problem solving, affect regulation and organization within the family. Number of sessions vary, depending on the adolescent's progress in resolving 5 specific tasks, and is delivered by a therapist trained in ABFT.

Supplements routine care by facilitating weekly contact between adolescents and their chosen support person (outside of the family), based on the notion that support people may minimize the impact of negative family environment. Support is provided to their adolescent by their nominated individual, who is asked to be in weekly contact with the adolescent. Support personnel are given $1.5-2 \mathrm{~h}$ of training.

Similar to YST-I, but with updated psychoeducation materials and the requirement that the nominated support person be an adult (rather than a peer). The support person has weekly check-ins with the adolescents for 3 months following hospitalization.

Pineda and Dadds (41) Strengths-based family psycho-education program, enhancing understanding of SH and SA, along with strategies to help minimize future self-injurious behavior, and information to facilitate access to support services. Sessions $2 \mathrm{~h}$ and held once a week or fortnightly. 
Dialectical Behavioral Therapy for Adolescents, (DBT-A) addresses self-driven cognitive-behavioral and regulatory processes (e.g., emotion regulation, distress tolerance, and interpersonal effectiveness) and the social environment through inclusion of a weekly multi-family skills training group and as needed family therapy sessions. A first RCT evaluating DBT-A was conducted in Norwegian clinics and recruited youths with both at least two episodes of self-harm and symptoms of borderline personality disorder (43). This trial compared DBT-A to treatment as usual enhanced by a therapist agreement to provide at least 1 weekly session during the trial. Results indicated that the 19-weeks DBT-A reduced the frequency of self-harm with large effect sizes, compared to moderate and weak effect sizes in the TAU condition (29). The advantage of DBT-A for reducing self-harm extended to a 1-year post-treatment follow-up, with DBT-A youths continuing to demonstrate fewer episodes of self-harm compared to treatment as usual youths (42). The authors looked at a range of additional clinical outcomes including suicidal ideation, hopelessness, depression, borderline symptoms, and global functioning. Results indicated that while there was an initial advantage for DBT-A and DBT-A youths continued to show improved clinical and functioning outcomes at the 1-year post-treatment follow-up, with time the TAU youths caught up and looked similar to the DBT-A youths on these more general outcomes. This trial was not powered to evaluate outcomes regarding suicide attempts, thus such data were not reported.

The combined self-driven and socially-driven intervention model was used in two U.S. treatment development trials. Both trials used a 2-therapist model with one therapist focusing on the youth and the other the parent, and both studies showed evidence of benefits in reducing the risk of suicide attempts. First, Esposito-Smythers et al. (28) tested an integrated CBT (ICBT) protocol for suicidality (along with co-occurring alcohol and drug related problems) and found that those randomized to I-CBT had fewer suicide attempts over the course of 18-months compared to those in the control condition. Second, Asarnow et al. (31) developed a DBT-informed cognitive-behavioral family treatment (referred to as SAFETY) which included attention to strengthening self-driven cognitive, behavioral and regulatory processes in the youth and parents, and family sessions aimed at promoting increased support and protection within the family and broader social environment. Results of this trial indicated a statistically significant advantage for SAFETY in decreasing suicide attempts over the 3-month treatment period, and reducing the risk of a first incident suicide attempt over a 6 to 12-month follow-up period. Weaker non-significant group differences were found for non-suicidal self-injury. While results of these trials are encouraging, it should be noted that both studies were relatively small treatment development trials, underscoring the need for cautious interpretation until replication is achieved.

\section{Overall Group Differences Irrespective of Therapeutic Intervention}

Overall symptom reduction across both treatment and control groups was found throughout several other studies included in this review, not specific to intervention type, including the Green et al. (23) trial of Developmental Group Psychotherapy intervention. In a specific clinical sample of Borderline Personality Disorder (BPD), three studies assessed the efficacy of cognitively-informed interventions. Chanen et al. (33) and Schuppert et al. $(25,26)$ investigated the efficacy of Cognitive Analytic Therapy (CAT) and Emotion Regulation Training (ERT), respectively, against TAU. Both CAT and ERT emphasize ways to react and respond to stressful situations with tools to more effectively manage stressful situations with aspects of selfdriven processes. Both studies only observed the reduction of self-harm within the whole cohort with no statistically significant group differences, indicating that these therapies do not appear to perform any better than TAU in reducing self-harm within Borderline patients.

\section{Null or Negative Findings}

Several interventions included in this update demonstrated non-significant or negative findings. For instance, The YouthNominated Support Treatment (YST-I) is a socially driven treatment that focuses on improving support in youths' social support network and is added to TAU, with version II of the intervention focused on strengthening social support in youthnominated supportive adults, rather than adults and peers. In both YST-I (39) and YST-II (40), no significant treatment effect was found in reduction of SA; authors emphasized the need for further research using this mode.

Cotgrove et al. (35) demonstrated non-significant findings regarding secondary prevention of suicide attempts in adolescents, examining re-admissions to Emergency Rooms. Adolescents were randomly allocated to a group receiving tokens guaranteeing re-admission to emergency services if they felt unable to cope within their environment. While there was no significant difference noted between adolescents with tokens and those without, those in the treatment condition had fewer repeat attempts than the control group, suggesting possible efficacy of a secondary prevention mechanism.

Two studies identified advantages of brief mental health interventions for linking youths to outpatient treatment after emergency presentation for suicidality and/or self-harm. Asarnow et al. (6) looked at a brief, cognitive-behavioral familybased Emergency Department (ED) intervention and found an advantage of the this intervention compared to treatment as usual for establishing linkage to outpatient care (the primary study outcome). Clinical outcomes were not evaluated close in time to the Emergency Department intervention. However, when clinical outcomes were evaluated roughly 2 months after discharge from the Emergency Department, no statistically significant advantage was found for this intervention in reducing suicide attempts. Importantly, there was also no evidence that linkage to outpatient treatment as usual after discharge from emergency services had any advantages relative to no post-discharge community treatment as usual.

Ougrin et al. (34) applied a brief therapeutic intervention incorporating elements of cognitive analytic therapy called Therapeutic Assessment (TA) following emergency presentation of self-harm. While there was no significant difference in the 
TABLE 2 | Types of Therapeutic Intervention for the Selected Randomized Controlled Trials and Aspects of Individual or Social Components.

\begin{tabular}{|c|c|c|c|c|c|c|c|c|c|}
\hline & \multicolumn{5}{|c|}{ Self-driven Components } & \multicolumn{4}{|c|}{ Social Components } \\
\hline & CBT & DBT & MBT & CAT & $\begin{array}{l}\text { Problem } \\
\text { Solving }\end{array}$ & $\begin{array}{l}\text { Social } \\
\text { Support }\end{array}$ & $\begin{array}{c}\text { Family } \\
\text { Involvement }\end{array}$ & $\begin{array}{l}\text { Psycho- } \\
\text { education }\end{array}$ & $\begin{array}{c}\text { Communication } \\
\text { skills }\end{array}$ \\
\hline Alavi et al. (27) & $\bullet$ & & & & & & & & \\
\hline Asarnow et al. (6) & & & & & & & $\bullet$ & & \\
\hline Asarnow et al. (31) & $\bullet$ & $\bullet$ & & & & & $\bullet$ & & \\
\hline \multicolumn{10}{|l|}{ Cotgrove et al. (35) } \\
\hline Chanen et al. (33) & & & & $\bullet$ & & & & & \\
\hline Diamond et al. (38) & & & & & & & $\bullet$ & & \\
\hline Diamond et al. (30) & $\bullet$ & & & & $\bullet$ & & $\bullet$ & & \\
\hline Esposito-Smythers et al. (28) & $\bullet$ & & & & & & $\bullet$ & & $\bullet$ \\
\hline Green et al. (23) & $\bullet$ & $\bullet$ & & & & & $\bullet$ & & $\bullet$ \\
\hline Harrington et al. (36) & & & & & & & $\bullet$ & & \\
\hline Hazell et al. (22) & $\bullet$ & $\bullet$ & & & $\bullet$ & & & & $\bullet$ \\
\hline King et al. (39) & & & & & & $\bullet$ & & $\bullet$ & \\
\hline King et al. (40) & & & & & & $\bullet$ & & $\bullet$ & \\
\hline Mehlum et al. (42) & & $\bullet$ & & & & & $\bullet$ & & $\bullet$ \\
\hline Ougrin et al. (34) & & & & $\bullet$ & & & $\bullet$ & & \\
\hline Pineda and Dadds (41) & & & & & & & $\bullet$ & $\bullet$ & \\
\hline Rossouw et al. (32) & & & $\bullet$ & & & & $\bullet$ & & \\
\hline Schuppert et al. (26) & & $\bullet$ & & & & & & & \\
\hline Schuppert et al. (25) & & $\bullet$ & & & & & & & \\
\hline Wharff et al. (37) & $\bullet$ & & & & & & $\bullet$ & & \\
\hline Wood et al. (24) & $\bullet$ & $\bullet$ & & & $\bullet$ & & & & $\bullet$ \\
\hline
\end{tabular}

frequency of self-harm resulting in emergency presentations between the Therapeutic Assessment and treatment as usual groups, overall treatment engagement remained higher in the Therapeutic Assessment group than the control group. Collectively, the Asarnow et al. \& Ougrin et al. studies underscore the value of brief mental health interventions for improving linkage to outpatient treatment after emergency presentation for self-harm and suicidality, as well as the importance of efforts to identify effective treatment strategies and implement them effectively within community programs.

\section{Secondary Outcome Assessment: Suicidal Ideation, Depression, Other Clinical, and Functioning Outcomes} Significant Differences in Therapeutic Interventions vs. Treatment as Usual

Of the possible 21 studies, 17 studies explored secondary outcomes of depressive symptoms and suicidal ideation, of which $5(29.4 \%)$ yielded significant differences by the intervention. Beginning with studies using primarily self-driven interventions, MBT-A was found to yield a significant reduction in depressive symptoms at the 12-month point (32). Both the treatment and control group showed reduced depressive symptoms, and a significant reduction over time was seen only in MBT-A youths condition. The largest mean difference between groups was seen at 9 months. Using a 12 -week treatment period, Alavi et al. (27) evaluated the CBT-Suicide Prevention (CBT-SP) treatment developed for the Treatment of Adolescent Suicide Attempter's (TASA) study (44), results indicated significant reductions in both suicidal ideation and depressive symptoms at the end of the 3-month treatment period. These results are similar to those from the TASA trial, which was originally designed as an RCT but random assignment was discontinued due to patients' reluctance to accept randomization. The TASA trial found relatively low rates of suicidal events, including suicide attempts, interrupted suicide attempts, and levels of suicidal ideation requiring emergency evaluation or hospitalization (19) as well as declines in suicidal ideation and depressive symptoms over the 6-month treatment period (45).

For interventions focusing on social support and psychoeducation strategies, King's investigations of both versions of the Youth-Nominated Support Team intervention decreased suicidal ideation over time in both groups. Further, the first version of the intervention, YST-I, allowed both youth-nominated peers and adults in the Support Team and demonstrated a small to medium effect on the reduction of suicidal ideation only after altering analyses from intent-totreat to actually treated; an effect that was seen only in female participants (39). Following with a study of the adapted YST-II intervention, which focused on youth-nominated adults, King again demonstrated a small to medium effect in the reduction of suicidal ideation, this time, only in those participants who had a history of multiple suicide attempts (40).

Another socially-driven intervention, Attachment-Based Family Therapy (ABFT), compared to TAU, was shown to 


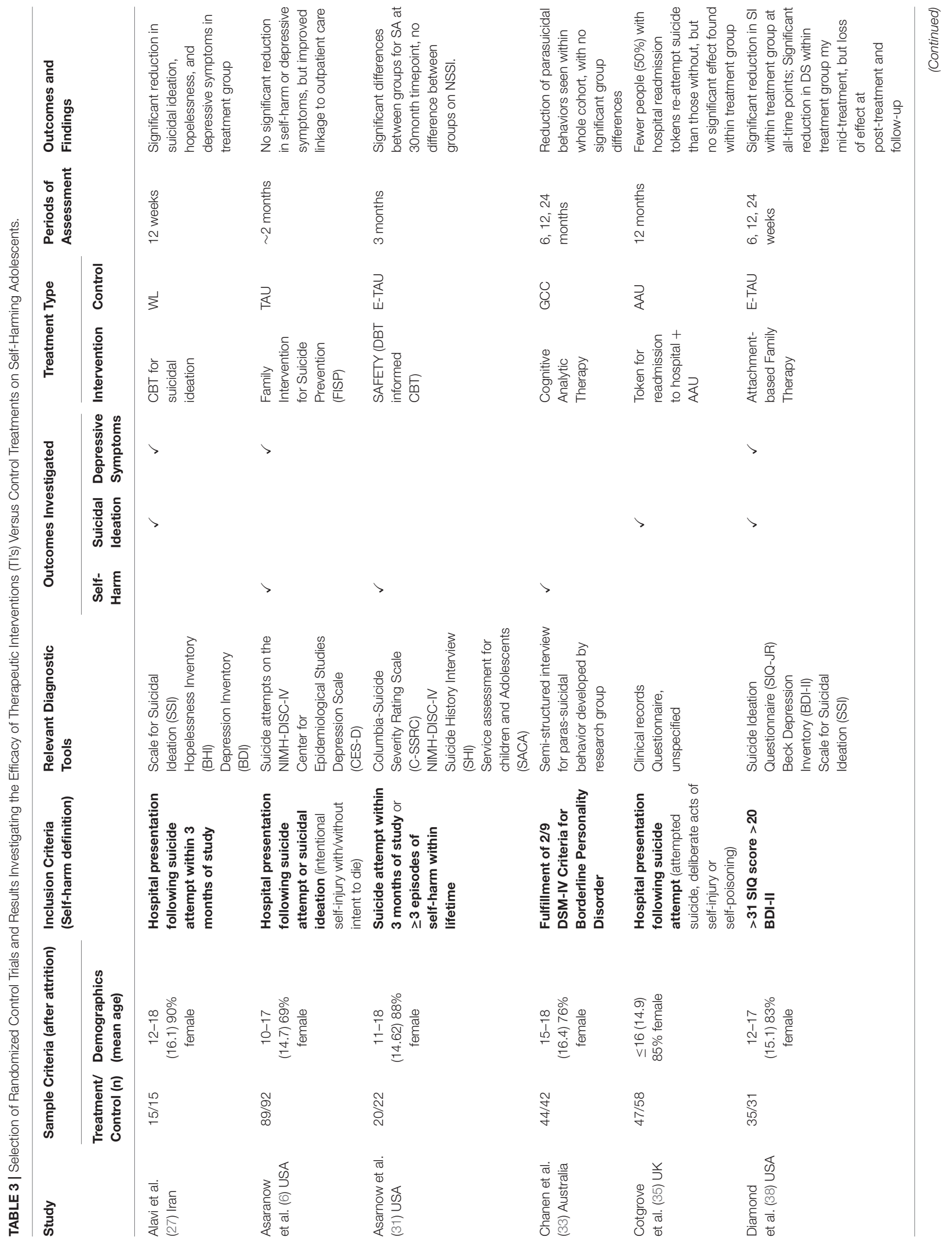




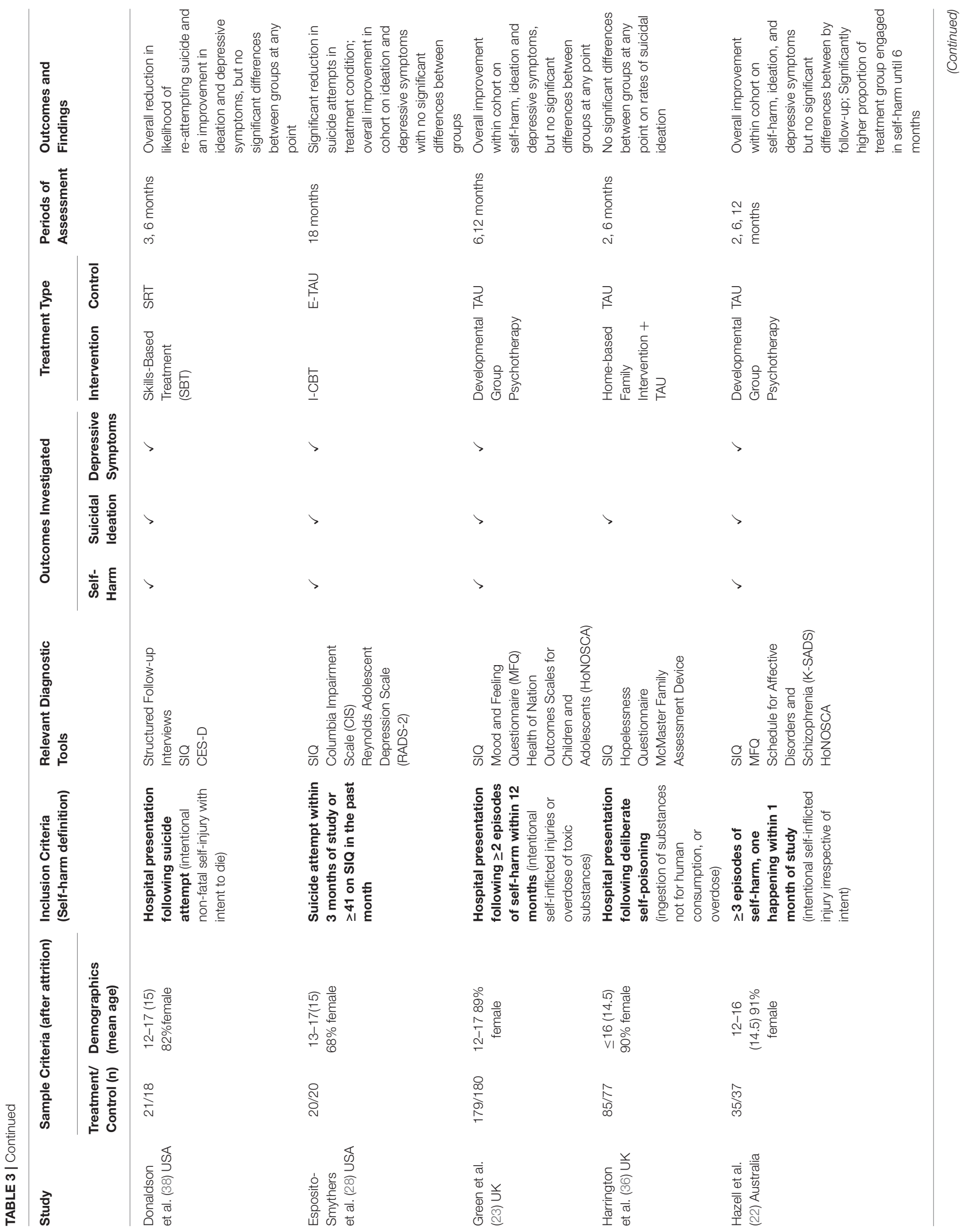




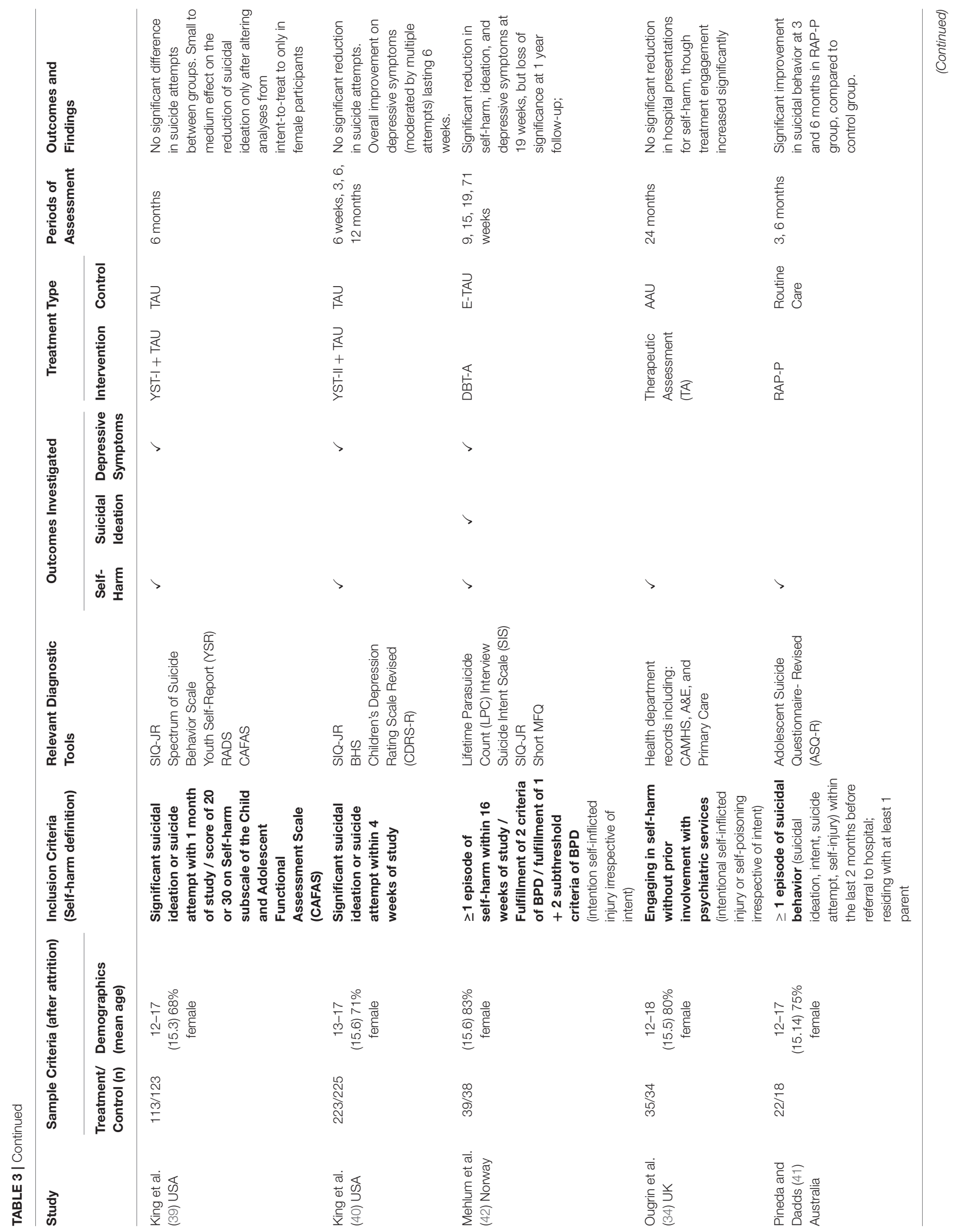




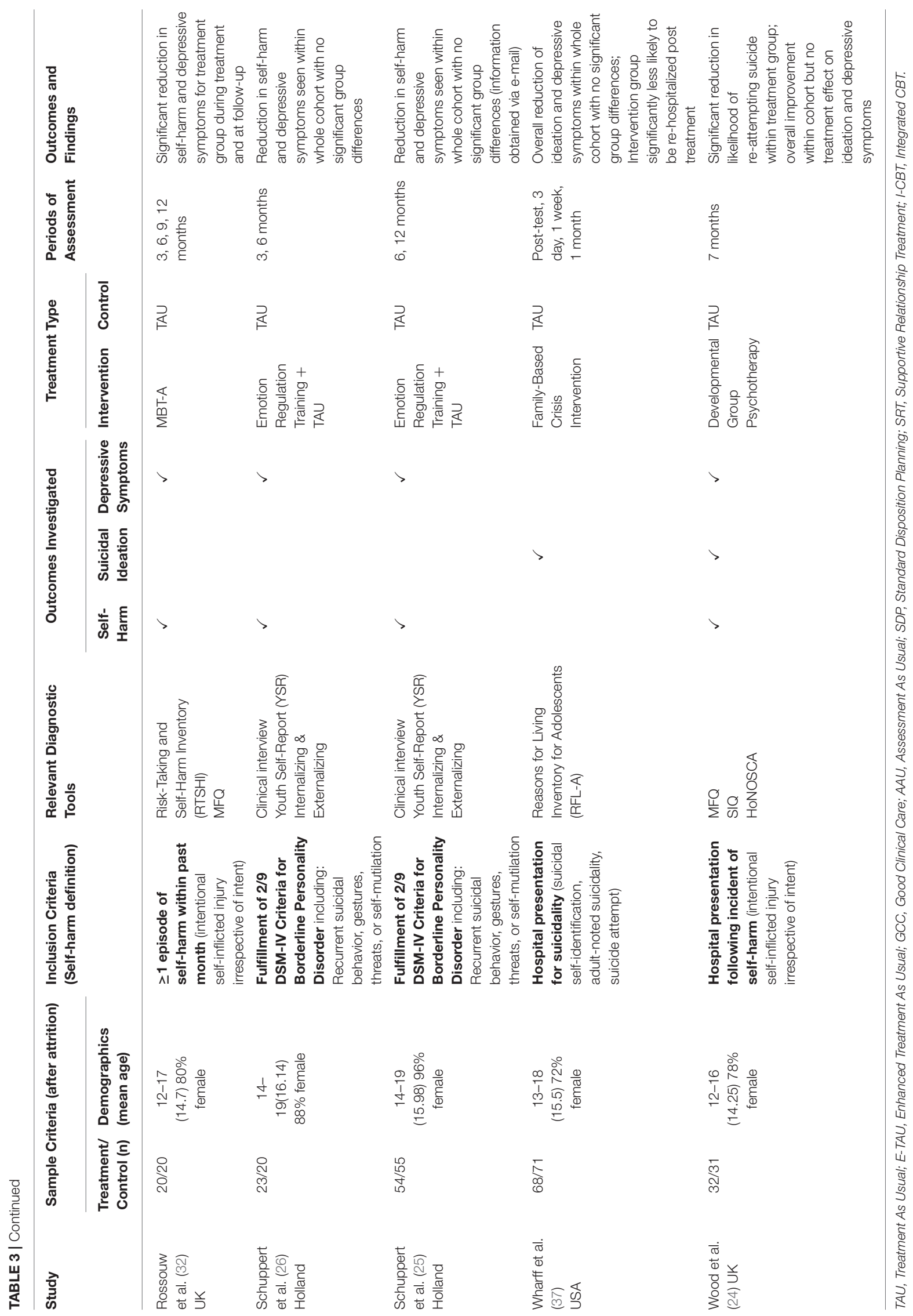


have very strong effects in reducing suicidal ideation during all points of treatment with the strongest effect observed at 24 weeks, the final follow-up (38). This effect was seen within the total sample, and within a subsample of adolescents who met the diagnostic criteria for clinical depression. Additionally, a significant effect in the reduction of suicidal ideation was seen within the Home-Based Family intervention, but only when controlling for depression.

\section{Overall Group Differences Irrespective of Therapeutic Intervention}

Reductions over time in suicidal ideation were observed in nearly all studies, including those evaluating mentalization treatment, DBT-A, integrated CBT for suicidality and substance abuse, skills based treatment, youth-nominated support teams, and attachment based family treatment (28-30, 32, 38).

While a significant reduction in ideation was observed in 3 studies based on social or family models (38-40), none of these studies identified significant differences in the reduction of depressive symptoms between treatment and control groups at the final follow-up measure. The Attachment-Based Family therapy intervention appeared close to producing a nearly significant result at the 6 and 12-week measurements, but was unable to reach statistical significance altogether at the end of the trial (38).

When examining socially-driven interventions within an emergency service settings, Asarnow et al. (6) did not examine suicidal ideation or depressive symptoms close in time to the emergency intervention. However, when followed up roughly 2months post -hospital discharge there were significant declines in depressive symptoms across groups. Of the socially-driven interventions administered during a presentation to emergency services or at the time of a psychiatric assessment, only Wharff investigated whether a Family-Based Crisis Intervention, a multimodule single session intervention would impact the adolescent's suicidal ideation (46). While an overall reduction in suicidal ideation was seen within the cohort, there was no significant treatment effect. In contrast to the other studies included this review, Wharff conducted pre-and post-test measures a mere 4-h apart.

\section{Null or Negative Findings}

Despite noting significant group differences in primary outcomes, Wood et al. (24) found no significant effect of Developmental Group Psychotherapy on reducing depressive symptoms and suicidal ideation. Consistent with what was noted for primary outcomes, neither of the two additional trials evaluating the same treatment $(22,23)$ saw treatment effects in secondary outcomes of reducing suicidal ideation or depressive symptoms.

Asarnow et al. (6) found no statistically significant advantage of the Emergency intervention in reducing depression levels, even though the intervention was associated with improved linkage to outpatient mental health services was observed. Indeed, there was no evidence that attendance in outpatient community treatment as usual was associated with lower depressive symptoms or suicidality.

\section{DISCUSSION}

We set out to examine the available literature for adolescents with a recent history of self-harm or suicide attempt, with the overall aim of clarifying which therapeutic interventions and approaches show evidence of benefits for reducing self-harm, suicide attempts, as well as suicidal ideation and depressive symptoms. Of the 18 unique interventions identified through this review, treatments that target individual, self-driven (cognitive-behavioral, selfregulatory processes) and socially-driven (family or social support network) processes appeared to show the greatest promise for reducing suicide attempts $(28,31)$, and there are data supporting the benefits of DBT-A and MBT-A (combined self-driven and systems-driven approaches), for reducing overall self-harm. If the somewhat different variations of CBT are considered together, and DBT-A is classified as a type of CBT, CBT is the only intervention type where initial positive findings have been replicated independently. It should be noted, however, that all of the CBT interventions with evidence for efficacy have strong family systems-driven components (I-CBT, SAFETY, DBTA). Other interventions with initial positive outcomes, such as MBT-A require testing in adequately powered trials and replication.

The results of this review update are demonstrative of the effectiveness of DBT-A and CBT, the only interventions where initial positive findings have been replicated independently. These interventions are therefore an invaluable part of the clinical treatment of young people who present with self-harm and a history of suicide attempts.

Turning to the secondary outcomes of suicidal ideation and general measures of suicide risk, current research supports the efficacy of attachment based family treatment and the Resourceful Adolescent Parent Program. Results were not reported on suicide attempts specifically or self-harm in either trial, and replication is needed. While not all studies have reported on suicidal ideation when suicide attempts and self-harm were primary outcomes, both MBT-A and DBTA have shown significant advantages in reducing both selfharm and suicidal ideation. Rossouw and Fonagy (32) reported an advantage for MBT-A compared to treatment as usual at end of the year-long treatment. Mehlum et al. (42) similarly reported an advantage for DBT relative to treatment as usual at end of treatment, which was at 16 weeks, although treatment as usual youths had caught up with the DBT-A youths by 71-weeks.

Results on depression outcomes tended to be similar to those for suicidal ideation, with a tendency for depression levels to diminish over time, and between group differences observed in the studies and time points where benefits on suicidal ideation were observed. The two studies that evaluated borderline symptoms (DBT-A and MBT-A) reported intervention benefits in reducing symptoms of Borderline Personality Disorder. Both suicidal ideation and depressive symptoms have been shown to precipitate non-suicidal self-injury and suicide attempts 
in adolescents $(19,47)$, and thus should not be overlooked as integral symptoms to address during treatment in future research.

Overall, the studies which showed significant effects in the reduction of outcomes at any point during treatment (without adjustments) were similar in several characteristics. First, these interventions mandated the family or support person's involvement in the adolescent's therapeutic journey in addition to the adolescent's individual therapy; five interventions included parental involvement throughout the duration of the intervention, through family training, family therapy, and/or family planning (28, 29, 31, 32, 38, 41). The Responsible Adolescent Parenting Program (2013) was the only exclusively socially-driven parenting intervention that yielded significant effects, suggesting a need for replication studies using this treatment. Finally, we noted that the effective interventions all share emotion regulation, problem solving, and communication skills as key tenets of the intervention. While dysregulated affect is shown to be a predictor of suicide attempts and non-suicidal selfinjury (47), subsequent research is needed to assess whether problem-solving and communication skills would contribute to the reduction of self-harm, suicidal ideation, or depressive symptoms.

The current National Institute for Health and Care Excellence (NICE) guidelines (48) for the treatment of adolescents with self-harm, suggest tailored treatments incorporating elements of cognitive behavioral, psychodynamic and problem-solving therapies. Results of this review are generally consistent with these guidelines, and clinical guidance is needed to support optimal clinical care for this potentially life-threatening problem. However, it is important to note that the evidence is limited. We still lack replicated evidence of treatment efficacy for any of the reviewed interventions. It is also important to note that the sampling protocols and populations differed across studies, and these sampling differences could lead to differences in treatment efficacy and study results. For instance, the DBT-A trial selected youths based on the presence of repeated self-harm and symptoms of borderline personality disorder, the MBT-A trial selected youths for the presence of self-harm within the past month, the Integrated CBT model recruited youths with both suicidality (attempts or ideation) and substance abuse, the SAFETY trial selected youths based on the presence of suicide attempts or repeated self-harm, and the Peer Nominated Support Team trials recruited youths with previous suicide attempts or suicidal ideation. Because trials were conducted across different nations and health systems, "treatment as usual" will have varied considerably, and this could conceivably have affected the observed between group differences. Further, with few exceptions $(32,41)$ studies have not yet reported on treatment mediators and studies aimed at treatment mechanisms associated with reduced fatal and non-fatal self-harm risk would help guide the field. Research focusing on targeted interventions such as those aimed at reducing access to dangerous methods of self-harm (firearms, poisoning) could also help inform clinical care and data are accumulating supporting the value of such interventions.

\section{Strengths and Limitations}

The current review supplements the literature by conducting a systematic review on not only suicide attempts and selfharm, but also addresses the links between suicidal ideation and depressive symptoms in adolescents. While we were able to add a more comprehensive component to the systematic review, due to the complexity of the varied studies and primary and secondary outcomes, additional research is needed to identify effective treatment strategies, provide guidance regarding how to best personalize treatment and match youths and families to treatments that are most likely to be beneficial, and to develop cost-effective treatment delivery strategies. There are no published RCTs of pharmacological interventions for the reduction of self-harm. However, many young people who selfharm are offered pharmacological treatment to address cooccurring psychiatric symptoms. While an investigation of the possible influence of pharmacological interventions was out of the scope of the current review, future work addressing both psychosocial and psychopharmacological elements of treatment is needed. To date, there have been no RCTs evaluating the efficacy of psychopharmacological treatments for reducing selfharm, though this may also be a potential avenue for future research. In following the methodology of the previous analysis, studies which did not adhere to an RCT design were excluded, though may nonetheless be insightful in regards to reducing self-harm in young people. Additionally, as the search terms used to identify studies on self-harm were derived from the subject headings of the relevant databases and were limited to studies published in English, some studies representing unique cultural outlooks on self-harm may have been omitted. Indeed, the heterogeneity of the studies included in this review makes it difficult to account for additional influential factors, including the participants' previous engagement with therapy, their mental health histories and psychiatric co-morbidities, and the quantity and severity of their previous self-harm, among others. Future reviews may wish to pay particular attention to such elements in order to understand whether certain interventions work more effectively in some unique cases over others. A variety of additional factors, including small and highly selective samples limit the generalizability of the findings in some of the studies included in this review. Finally, the current failure to replicate certain interventions in different cultural contexts underscores the challenges for exporting treatment strategies across different cultures and settings, and the importance of building international consensus and developing care strategies that can work across diverse cultural contexts and health systems.

\section{Future Directions for Effective Interventions}

Considering future directions for studies aimed at decreasing self-harm and suicide attempts in adolescents, some treatments (such as DBT-A) led to significant reductions on outcomes more rapidly than others. Cost analyses could further inform knowledge about the viability of delivering certain interventions over others in routine clinical settings to large populations. Several of the evaluated interventions require multiple personnel 
(e.g., 2-therapist model used in the Integrated CBT model, the SAFETY intervention, and DBT-A with skills trainer and individual therapist). Likewise, whereas several of the interventions are completed in under 6 months, others require long and perhaps costly commitments to therapy, which may act as barriers to treatment adherence, particularly for adolescents who experience a lack of motivation to attend sessions as a symptom of depression $(49,50)$. These cost considerations will also have a major bearing on the likelihood of interventions being implemented in routine health setting. Additionally, some of the multi-component treatments, such as DBT-A which requires $1 \mathrm{~h}$ weekly psychotherapy plus $2 \mathrm{~h}$ of multi-family skills group may require a more intensive and burdensome treatment dose than needed for some youths and families. Stepped care approaches that match treatment intensity to assessed level of risk and need may prove helpful for identifying the most cost-effective treatment delivery strategies.

The results of a large, multi-center RCT investigating the effectiveness of Family Therapy in reducing self-harm in adolescents (51) were published following the intial literature review, and thus cannot be included in the results of the current update. However, these results, which demonstrated that Family Therapy was more costly and no more beneficial than treatment as usual, are nonetheless an important contribution to understanding which interventions are viable and effective in reducing self-harm in young people. In comparison to other therapies which include a strong family-based component as part of the treatment (such as DBT-A, MBT-A, and RAP-P among others), participants in the Family Therapy group were provided with 6-8 monthly sessions of therapy; far fewer than those participants which received any of the aforementioned treatments, indicating that the duration and intensity of the treatment may be an important factor in the success of an intervention aiming to reduce self-harm and preventing suicide attempts.

Other RCTs published following the completion of our search, include a study further strengthening the efficacy of DBT-A in reducing self-harm post-treatment, as well as a significantly lower number of suicide attempts and significantly fewer episodes of non-suicidal self-injurious behavior. Although no significant between-group differences were found at longer-term (12month) follow-up in number of self-harm episodes, youths in the DBT-A were significantly more likely to show clinically significant change, defined as the absence of any self-harm, through the 12-month follow-up (52). Another study showed that young people with longer inpatient admissions were more likely to have multiple self-harm episodes, than young people treated with intensive community care (53).

Lastly, there were several unpublished protocol studies of RCTs for this subject population (54-58) and studies in progress

\section{REFERENCES}

1. Prevention NAAFS. (2014). Research Prioritization Task Force. A Prioritized Research Agenda for Suicide Prevention: An Action Plan to Save Lives.
(59-61), which we were unable to use for our systematic review. It is our hope that when these protocols are applied and published, such findings will greatly advance the field, and shed further light on effective treatments for adolescents at-risk for suicide.

Our updated systematic review suggests that given the heterogeneity of suicidal behavior, understanding which type of intervention is most effective for adolescents at risk of suicide can be a challenging but nonetheless paramount endeavor that requires further attention.

\section{AUTHOR CONTRIBUTIONS}

UI and NS conducted the update to the systematic review, including the literature search, and analysis, UI and NS wrote the draft of the paper, JRA, PM, and TT added clinical and critical insight to the overall paper structure, and DO supervised the procedure and the overall study.

\section{FUNDING}

The authors' time was paid for by Kings College London and South London and Maudsley NHS Foundation Trust.

JRA has received grant, research, or other support from the National Institute of Mental Health, the American Foundation for Suicide Prevention, the Substance Abuse and Mental Health Services Administration, the American Psychological Association (APA), the Society of Clinical Child and Adolescent Psychology (Division 53 of the APA), and the Association for Child and Adolescent Mental Health. She has served as a consultant on quality improvement for depression and suicide/self-harm prevention, serves on the Scientific Council of the American Foundation for Suicide Prevention, and the Scientific Advisory Board of the Klingenstein Third Generation Foundation.

PM is a co-applicant to the following grant: Mars B, Gunnell D, Joinson C, Moran P, Relton C, Hemani G, Heron J, Suderman M, Ford T. Pathways to self-harm: Biological mechanisms and genetic contribution. MRC. \$297,867. 2017-2019 Co-applicant (to PM).

\section{ACKNOWLEDGMENTS}

The authors thank Dr. Emily Simonoff, Dr. Bruce Clarke and Mrs. Jo Fletcher who made this work possible. This study was supported by the NIHR Biomedical Research Centre at University Hospitals Bristol NHS Foundation Trust and the University of Bristol. The views expressed in this publication are those of the author(s) and not necessarily those of the NHS, the National Institute for Health Research or the Department of Health and Social Care.
Rockville, MD: National Institute of Mental Health and Research Prioritization Task Force.

2. Patton GC, Coffey C, Sawyer SM, Viner RM, Haller DM, Bose K, et al. Global patterns of mortality in young people: a systematic analysis of population 
health data. Lancet (2009) 374:881-92. doi: 10.1016/S0140-6736(09) 60741-8

3. Butler AM, Malone K. Attempted suicide v. non-suicidal self-injury: behaviour, syndrome or diagnosis? Br J Psychiatry (2013) 202:324-5. doi: 10.1192/bjp.bp.112.113506

4. American Psychiatric Association. (2013). Diagnostic and Statistical Manual of Mental Disorders, 5th edn. Arlington, VA: American Psychiatric Publishing.

5. Hawton K, Harriss L. Deliberate self-harm in young people: characteristics and subsequent mortality in a 20 -year cohort of patients presenting to hospital. J Clin Psychiatry (2007) 68:1574-83. doi: 10.4088/JCP.v68n1017

6. Asarnow JR, Porta G, Spirito A, Emslie G, Clarke G, Wagner KD, et al. Suicide attempts and nonsuicidal self-injury in the treatment of resistant depression in adolescents: findings from the TORDIA study. J Am Acad Child Adoles Psychiatry (2011) 50:772-81. doi: 10.1016/j.jaac.2011.04.003

7. Brent DA, Mcmakin DL, Kennard BD, Goldstein TR, Mayes TL, Douaihy AB. Protecting adolescents from self-harm: a critical review of intervention studies. J Am Acad Child Adoles Psychiatry (2013) 52:1260-71. doi: 10.1016/j.jaac.2013.09.009

8. Ougrin D, Boege I. Brief report: the Self Harm Questionnaire: a new tool designed to improve identification of self harm in adolescents. J Adoles. (2013) 36:221-5. doi: 10.1016/j.adolescence.2012.09.006

9. Moran P, Coffey C, Romaniuk H, Olsson C, Borschmann R, Carlin JB, et al. The natural history of self-harm from adolescence to young adulthood: a population-based cohort study. Lancet (2012) 379:236-43. doi: 10.1016/S0140-6736(11)61141-0

10. Rowe SL, French RS, Henderson C, Ougrin D, Slade M, Moran P. Helpseeking behaviour and adolescent self-harm: a systematic review. Aust N Zeal J Psychiatry (2014) 48:1083-95. doi: 10.1177/0004867414555718

11. Ougrin D, Tranah T, Stahl D, Moran P, Asarnow JR. Therapeutic interventions for suicide attempts and self-harm in adolescents: systematic review and meta-analysis. J Am Acad Child Adoles Psychiatry (2015) 54:97-107.e102. doi: 10.1016/j.jaac.2014.10.009

12. Shaffer D, Gould MS, Fisher P, Trautman P, Moreau D, Kleinman M, et al. Psychiatric diagnosis in child and adolescent suicide. Arch Gener Psychiatry (1996) 53:339-48. doi: 10.1001/archpsyc.1996.01830040075012

13. Gould MS, King R, Greenwald S, Fisher P, Schwab-Stone M, Kramer R, et al. Psychopathology associated with suicidal ideation and attempts among children and adolescents. J Am Acad Child Adoles Psychiatry (1998) 37:91523. doi: 10.1097/00004583-199809000-00011

14. National Confidential Inquiry into Suicide and Homicide by People with Mental Illness. (2017). Suicide by Children and Young People. Manchester: University of Manchester, 2017.

15. Lundh L-G, Wångby-Lundh $\mathrm{M}$, Paaske $\mathrm{M}$, Ingesson $\mathrm{S}$, Bjärehed $\mathrm{J}$. Depressive symptoms and deliberate self-harm in a community sample of adolescents: a prospective study. Depress Res Treat. (2011) 2011:935871. doi: $10.1155 / 2011 / 935871$

16. Singhal A, Ross J, Seminog O, Hawton K, Goldacre MJ. Risk of self-harm and suicide in people with specific psychiatric and physical disorders: comparisons between disorders using English national record linkage. J R Soc Med. (2014) 107:194-204. doi: 10.1177/0141076814522033

17. Yang F-Y, Lai CY, Yen C-F, Hsu Y-Y, Zauszniewski JA. The depressive symptoms, resourcefulness, and self-harm behaviors of adolescents. J Nurs Res. (2017) 25:41-9. doi: 10.1097/jnr.0000000000000127

18. Zubrick SR, Hafekost J, Johnson SE, Sawyer MG, Patton G, Lawrence D. The continuity and duration of depression and its relationship to non-suicidal selfharm and suicidal ideation and behavior in adolescents 12-17. J Affect Disord. (2017) 220:49-56. doi: 10.1016/j.jad.2017.05.050

19. Brent DA, Greenhill LL, Compton S, Emslie G, Wells K, Walkup JT, et al. The Treatment of Adolescent Suicide Attempters study (TASA): predictors of suicidal events in an open treatment trial. J Am Acad Child Adoles Psychiatry (2009) 48:987-96. doi: 10.1097/CHI.0b013e3181b5dbe4

20. Jadad AR, Moore RA, Carroll D, Jenkinson C, Reynolds DJM, Gavaghan DJ, et al. Assessing the quality of reports of randomized clinical trials: is blinding necessary? Control Clin Trials (1996) 17:1-12. doi: 10.1016/0197-2456(95)00134-4

21. Spirito A, Boergers J, Donaldson D, Bishop D, Lewander W. An intervention trial to improve adherence to community treatment by adolescents after a suicide attempt. J Am Acad Child Adolesc Psychiatry (2002) 41:435-42. doi: 10.1097/00004583-200204000-00016

22. Hazell PL, Martin G, Mcgill K, Kay T, Wood A, Trainor G, et al. Group therapy for repeated deliberate self-harm in adolescents: failure of replication of a randomized trial. J Am Acad Child Adolesc Psychiatry (2009) 48:662-70. doi: 10.1097/CHI.0b013e3181a0acec

23. Green JM, Wood AJ, Kerfoot MJ, Trainor G, Roberts C, Rothwell J, et al. Group therapy for adolescents with repeated self harm: randomised controlled trial with economic evaluation. BMJ (2011) 2011:342. doi: 10.1136/bmj.d682

24. Wood A, Trainor G, Rothwell J, Moore A, Harrington R. Randomized trial of group therapy for repeated deliberate self-harm in adolescents. J Am Acad Child Adolesc Psychiatry (2001) 40:1246-53. doi: 10.1097/00004583-200111000-00003

25. Schuppert HM, Timmerman ME, Bloo J, Van Gemert TG, Wiersema $H M$, Minderaa RB, et al. Emotion regulation training for adolescents with borderline personality disorder traits: a randomized controlled trial. J Am Acad Child Adolesc Psychiatry (2012) 51:1314-1323.e1312. doi: 10.1016/j.jaac.2012.09.002

26. Schuppert HM, Giesen-Bloo J, Van Gemert TG, Wiersema HM, Minderaa RB, Emmelkamp PM, et al. Effectiveness of an emotion regulation group training for adolescents-a randomized controlled pilot study. Clin Psychol Psychother. (2009) 16:467-78. doi: 10.1002/cpp.637

27. Alavi A, Sharifi B, Ghanizadeh A, Dehbozorgi G. Effectiveness of cognitivebehavioral therapy in decreasing suicidal ideation and hopelessness of the adolescents with previous suicidal attempts. Iran J Pediatrics (2013) 23:46772. doi: 10.1016/j.neurenf.2012.04.266

28. Esposito-Smythers C, Spirito A, Kahler CW, Hunt J, Monti P. Treatment of co-occurring substance abuse and suicidality among adolescents: a randomized trial. J Consult Clin Psychol. (2011) 79:728-39. doi: $10.1037 / \mathrm{a} 0026074$

29. Mehlum L, Tormoen AJ, Ramberg M, Haga E, Diep LM, Laberg S, et al. Dialectical behavior therapy for adolescents with repeated suicidal and selfharming behavior: a randomized trial. J Am Acad Child Adolesc Psychiatry (2014) 53:1082-91. doi: 10.1016/j.jaac.2014.07.003

30. Donaldson D, Spirito A, Esposito-Smythers C. Treatment for adolescents following a suicide attempt: results of a pilot trial. J Am Acad Child Adolesc Psychiatry (2005) 44:113-20. doi: 10.1097/00004583-200502000-00003

31. Asarnow JR, Hughes JL, Babeva KN, Sugar CA. Cognitive-behavioral family treatment for suicide attempt prevention: a randomized controlled trial. I Am Acad Child Adolesc Psychiatry (2017) 56:506-14. doi: 10.1016/j.jaac.2017.03.015

32. Rossouw TI, Fonagy P. Mentalization-based treatment for self-harm in adolescents: a randomized controlled trial. J Am Acad Child Adolesc Psychiatry (2012) 51:1304-1313.e1303. doi: 10.1016/j.jaac.2012.09.018

33. Chanen AM, Jackson HJ, Mccutcheon LK, Jovev M, Dudgeon P, Yuen HP, et al. Early intervention for adolescents with borderline personality disorder using cognitive analytic therapy: randomised controlled trial. Br J Psychiatry (2008) 193:477-84. doi: 10.1192/bjp.bp.107.048934

34. Ougrin D, Boege I, Stahl D, Banarsee R, Taylor E. Randomised controlled trial of therapeutic assessment versus usual assessment in adolescents with self-harm: 2-year follow-up. Arch Dis Child. (2013) 98:772-6. doi: 10.1136/archdischild-2012-303200

35. Cotgrove A, Zirinsky L, Black D, Weston D. Secondary prevention of attempted suicide in adolescence. J Adoles. (1995) 18:569. doi: 10.1006/jado.1995.1039

36. Harrington R, Kerfoot M, Dyer E, McNiven F, Gill J, Harrington V, et al. Randomized trial of a home-based family intervention for children who have deliberately poisoned themselves. J Am Acad Child Adolesc Psychiatry (1998) 37:512-8.

37. Wharff EA, Ginnis KB, Ross AM, White EM, White MT, Forbes PW. FamilyBased Crisis Intervention With Suicidal Adolescents: A Randomized Clinical Trial. Pediatr Emerg Care (2017). doi: 10.1097/PEC.0000000000001076. [Epub ahead of print].

38. Diamond GS, Wintersteen MB, Brown GK, Diamond GM, Gallop R, Shelef K, et al. Attachment-based family therapy for adolescents with suicidal ideation: a randomized controlled trial. J Am Acad Child Adoles Psychiatry (2010) 49:122-31. doi: 10.1016/j.jaac.2009.11.002 
39. King CA, Kramer A, Preuss L, Kerr DC, Weisse L, Venkataraman S. Youth-nominated support team for suicidal adolescents (Version 1): a randomized controlled trial. J Consult Clin Psychol. (2006) 74:199-206. doi: 10.1037/0022-006X.74.1.199

40. King CA, Klaus N, Kramer A, Venkataraman S, Quinlan P, Gillespie B. The youth-nominated support team-version ii for suicidal adolescents: a randomized controlled intervention trial. J Consult Clin Psychol. (2009) 77:880. doi: $10.1037 / \mathrm{a} 0016552$

41. Pineda J, Dadds MR. Family intervention for adolescents with suicidal behavior: a randomized controlled trial and mediation analysis. J Am Acad Child Adoles Psychiatry (2013) 52:851-62. doi: 10.1016/j.jaac.2013.05.015

42. Mehlum L, Ramberg M, Tørmoen AJ, Haga E, Diep LM, Stanley BH, et al. Dialectical behavior therapy compared with enhanced usual care for adolescents with repeated suicidal and self-harming behavior: outcomes over a one-year follow-up. J Am Acad Child Adoles Psychiatry (2016) 55:295-300. doi: 10.1016/j.jaac.2016.01.005

43. Tørmoen A, Grøholt B, Haga E, Brager-Larsen A, Miller A, Walby F, et al. Feasibility of dialectical behavior therapy with suicidal and self-harming adolescents with multi-problems: training, adherence, and retention. Arch Suic Res. (2014) 18:432-44. doi: 10.1080/13811118.2013. 826156

44. Stanley B, Brown G, Brent DA, Wells K, Poling K, Curry J, et al. Cognitivebehavioral therapy for suicide prevention (CBT-SP): treatment model, feasibility, and acceptability. J Am Acad Child Adoles Psychiatry (2009) 48:1005-13. doi: 10.1097/CHI.0b013e3181b5dbfe

45. Vitiello B, Brent DA, Greenhill LL, Emslie G, Wells K, Walkup JT, et al. Depressive symptoms and clinical status during the Treatment of Adolescent Suicide Attempters (TASA) Study. J Am Acad Child Adoles Psychiatry (2009) 48:997-1004. doi: 10.1097/CHI.0b013e3181b5db66

46. Wharff EA, Ginnis KM, Ross AM. Family-based crisis intervention with suicidal adolescents in the emergency room: a pilot study. Soc Work (2012) 57:133-43. doi: 10.1093/sw/sws017

47. Wilkinson P, Kelvin R, Roberts C, Dubicka B, Goodyer I. Clinical and psychosocial predictors of suicide attempts and nonsuicidal self-injury in the Adolescent Depression Antidepressants and Psychotherapy Trial (ADAPT). Am J Psychiatry (2011) 168:495-501. doi: 10.1176/appi.ajp.2010.10050718

48. National Center for Clinical Excellence. (2011). Self-Harm in Over 8s: LongTerm Management. (Clinical Guideline CGI 33). Available online at: https:// www.nice.org.uk/guidance/cg133

49. Mohr DC, Ho J, Duffecy J, Baron KG, Lehman KA, Jin L, et al. Perceived barriers to psychological treatments and their relationship to depression. $J$ Clin Psychol (2010) 66:394-409. doi: 10.1002/jclp.20659

50. Sturm R, Sherbourne CD. Are barriers to mental health and substance abuse care still rising? J Behav Health Serv Res. (2001) 28:81. doi: $10.1007 / \mathrm{BF} 02287236$

51. Cottrell DJ, Wright-Hughes A, Collinson M, Boston P, Eisler I, Fortune $\mathrm{S}$, et al. Effectiveness of systemic family therapy versus treatment as usual for young people after self-harm: a pragmatic, phase 3, multicentre, randomised controlled trial. Lancet Psychiatry (2018) 5:203-16. doi: 10.1016/S2215-0366(18)30058-0

52. Mccauley E, Berk MS, Asarnow JR, Adrian M, Cohen J, Korslund K, et al. Efficacy of dialectical behavior therapy for adolescents at high risk for suicide: a randomized clinical trial. JAMA Psychiatry (2018) 75:777-85. doi: 10.1001/jamapsychiatry.2018.1109

53. Ougrin D, Corrigall R, Poole J, Zundel T, Sarhane M, Slater V, et al. Comparison of effectiveness and cost-effectiveness of an intensive community supported discharge service versus treatment as usual for adolescents with psychiatric emergencies: a randomised controlled trial. Lancet Psychiatry (2018) 5:477-85. doi: 10.1016/S2215-0366(18)30129-9

54. Rees CS, Hasking P, Breen LJ, Lipp OV, Mamotte C. Group mindfulness based cognitive therapy vs. group support for self-injury among young people: study protocol for a randomised controlled trial. BMC Psychiatry (2015) 15:154. doi: 10.1186/s12888-015-0527-5

55. Van Spijker BA, Calear AL, Batterham PJ, Mackinnon AJ, Gosling JA, Kerkhof AJ, et al. Reducing suicidal thoughts in the Australian general population through web-based self-help: study protocol for a randomized controlled trial. Trials (2015) 16:62. doi: 10.1186/s13063-015-0589-1

56. Wright-Hughes A, Graham E, Farrin A, Collinson M, Boston P, Eisler I, et al. Self-Harm Intervention: family Therapy (SHIFT), a study protocol for a randomised controlled trial of family therapy versus treatment as usual for young people seen after a second or subsequent episode of self-harm. Trials (2015) 16:501. doi: 10.1186/s13063-0151007-4

57. Haddock G, Davies L, Evans E, Emsley R, Gooding P, Heaney L, et al. Investigating the feasibility and acceptability of a cognitive behavioural suicide prevention therapy for people in acute psychiatric wards (the 'INSITE'trial): study protocol for a randomised controlled trial. Trials (2016) 17:79. doi: 10.1186/s13063-016-1192-9

58. Pepping CA, Lyons A, Mcnair R, Kirby JN, Petrocchi N, Gilbert P. A tailored compassion-focused therapy program for sexual minority young adults with depressive symotomatology: study protocol for a randomized controlled trial. BMC Psychol. (2017) 5:5. doi: 10.1186/s40359-0170175-2

59. Asarnow JR, and Clarke G. (2017). Randomized Controlled Trial of Stepped Care for Suicide Prevention in Adolescents and Young Adults. Available online at: https://clinicaltrials.gov: NIMH

60. Boudreaux E, and Kiefe C. (2017). A System of Safety: Preventing Suicided Through Healthcare System Transformation. Available online at: https:// clinicaltrials.gov

61. Kemp K. (2017). Implementing a Brief Suicide Intervention for High Risk Youth With Front-Line Juvenile Justice Staff. Available online at: https://clinicaltrials. gov

Conflict of Interest Statement: The authors declare that the research was conducted in the absence of any commercial or financial relationships that could be construed as a potential conflict of interest.

Copyright (c) 2018 Iyengar, Snowden, Asarnow, Moran, Tranah and Ougrin. This is an open-access article distributed under the terms of the Creative Commons Attribution License (CC BY). The use, distribution or reproduction in other forums is permitted, provided the original author(s) and the copyright owner(s) are credited and that the original publication in this journal is cited, in accordance with accepted academic practice. No use, distribution or reproduction is permitted which does not comply with these terms. 